\title{
Correlación manométrica, histológica e histoquímica en el estudio de megacolon en el niño
}

\author{
Dra. Tatiana Rudloff F., ${ }^{1}$ Dr. Guillermo Venegas Y., ${ }^{2}$ Dr. Ivan Bravo R., ${ }^{3}$ Dra. Karin Reinecke, ${ }^{4}$ Dra. Carmen G. \\ Acevedo P., ${ }^{3}$ Dr. Andrés Cantín D., ${ }^{5}$ Dr. Sergio Rojas C., ${ }^{6}$
}

Megacolon in children: Manometry, histology and histochemistry.

\begin{abstract}
Ninely-six anorectal manometric studies were performed in children with megacolon Sixteen patients had pressure records compatibles with Hirschsprung's disease: in twelve the rectal suction biopsy and study of acetylcholinesterase acbivity confirmed the diagnosis of aginglionosis.

In two pacients with non conclusive manometry, acetylchalinesterase activity was highly positive: in one the diagnosis of colonic neuronal dysplasia through the study of succinic dehydrogenase was confimed.
\end{abstract}

\section{INTRODUCCION}

En la prática pedićtrica diaria, con bastante frecuencia el médico se ve enfrentado a pacientes que consultan por dificultad en la evacuación intestinal. En un porcentaje no despreciable de ellos la consulta es suscitada por la preocupación excesiva de la familia sobre el control, naturaleza, tiempo y frecuencia de las deposiciones, y por el escaso conocimiento sobre lo que debe ser considerado normal o patológico. En los restantes o verdaderos casos de constipación, la causa radica fundamentalmente en la mala distribución de los componentes de la dieta, deficiente formación de hábitos, factores psicológicos, y en un grupo menor, en alteraciones orgánicus o métabólicas.

Si bien es cierto, que la elaboración de una historia clínica detallada, así como el tacto rectal y la exploración radiólogica permitirá diferenciar, en la mayoria de los casos, aquellos niños cuya constipación es atribuible a una causa funcional de la que obedece a una orgánica, existe siempre un número de pacientes cuya etiología no puede esclarecerse con precisión sin un estudio histológico que demuestre la normalidad o alteración de las estructuras orgánicas comprometidas en la función evacuadora del intestino. La biopsia es por lo tanto un paso imprescindible en el diagnóstico de megacolon, pero indiscutiblemente

\footnotetext{
${ }_{1}$ Médicn Pediatra, Servicio de Pediatria, Hospital Gmo. Grant B. Concepción.

2 Docente Depto. Pediatría, Universidad de Concepcion.

${ }^{3}$ Depto. Ciencias Fisiologicas, U, de Concepción.

${ }^{4}$ Depto. Histologia, U. de Concepción.

5 Depto. Hadiologia, Hospital G. Grant B. de Concepeion.

'́servicio Cingla Infantil, Hospital L. Mascayano, Concepción.
}

constituye un método agresivo, con potenciales peligros de perforación o estenosis residual.

Desde 1963 y basándose en los trabajos de Schuster, se emplea la técnica eletromanométrica en la investigación de la motilidad ano-rectal. Su valor en el diagnóstico de la enfermedad de Hirschsprung ha quedado fiuera de discusión después de los trabajos de Larson, Schnaufer y Meunier, quienes han demostrado una alteración en el comportamiento esfinteriano, específica de esta afección. ${ }^{1-10}$

La biopsia rectal por succión, que incluye exclusivamente mucosa y submucosa para visualizar el plexo de Meissner, se utiliza desde 1965, obviando así la mayoría de los inconvenientes de las biopsias de pared total. Se trata de un método no agresivo y que carece de complicaciones; sin embargo, la evaluación histológica del plexo subınucoso requiere gran experiencia. El hallazgo de un aumento significativo de la producción de acetilcolinesterasa a nivel de fibras adrenérgicas y colimérgicas en el recto aganglionar constituye la base de un método inocuo y altamente específico para certificar el diagnóstico de aganglionosis intestinal congénita. ${ }^{\mid 1-24}$

El objetivo del presente trabajo es evaluar el rendimiento de la manometría rectal en relación con el estudio histoquímico e histológico de la mucosa rectal, en niños portadores de megacolon, controlados en la Policlínica de Gastroenterología Infantil del Hospital Gmo. Grant Benavente de Concepción.

\section{MATERIAL Y METODO}

Entre los años 1978 y 1980 , se han practicado 96 manometrias rectales en niños con megacolon, 
agregándose desde 1979 la toma de biopsia rectal por succión en 24 de ellos.

Como grupo de control para las manometrías, se realizó el examen en 10 niños normales. Como grupo de control para las biopsias rectales por succión, se tomaron muestras de pared en 12 ninos operados de enfermedad de Hirschsprung.

La manometría rectal tiene como propósito evaJuar los fenómenos motores propios de esta región. Se realiza por medio de la técnica electromagnética de Meunier y Cols. modificada, que consiste en introducir en el canal anal, dos catéteres de polietileno por los cuales se perfunde liquido, registrando la presión generada por la mayor a menor resistencia al flujo del líquido a

Figura 1

ESFINT ER TMERTO
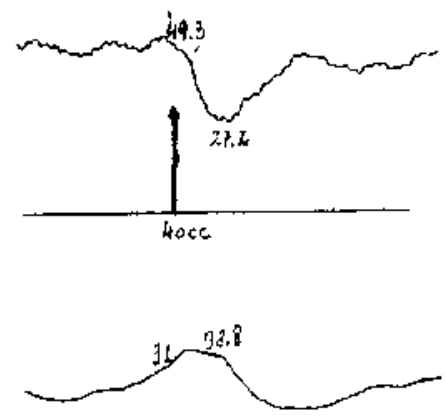

$\overbrace{\text { ESINTER ETTERO }}$

La biopsia rectal por succión tiene como finalidad el estudio de la actividad enzimática en el tejido intersticial de la mucosa. Se utiliza el Aparato de Ulrich tomando 2 muestras simultáneas a 5 y $6 \mathrm{~cm}$. del margen anal. Estas muestras se congelan con nieve carbónica, se almacenan a $-70^{\circ} \mathrm{C}$ y posteriormente se hacen cortes, en un criostato de 14 micrones de espesor que se tiñen con Hematoxilina-Eosina para verificar la presencia de epitelio, túnica propia y muscular de la mucosa, y luego, mediante el método de Karnowsky-Roots modificado, se determina actividad acetilcolinesterásica. nivel de los esfínteres intemo y extemo. Los canbios en la actividad esfinteriana son inducidos por la dilatación de la ampolla rectal mediante la insuflación de aire a un pequeño balón de látex introducido a $6-8 \mathrm{cms}$. del margen anal. Para el re gistro de las presiones se conectan los catéteres a transductores de presion Statham $y$ a un polígrafo Gilson multicanal. La dilatación del recto normal induce a la relajación del esfinter interno $y$ a la contracción del extemo.

En los pacientes con segmento agangliónico se detecta la ausencia de reflejo rectal, evidenciado por la falta de relajación del esfínter interno o una respuesta paradojal con contracción del estinter interno frente a la estimulacion. (Figuras 1 y 2 ).

Figura 2
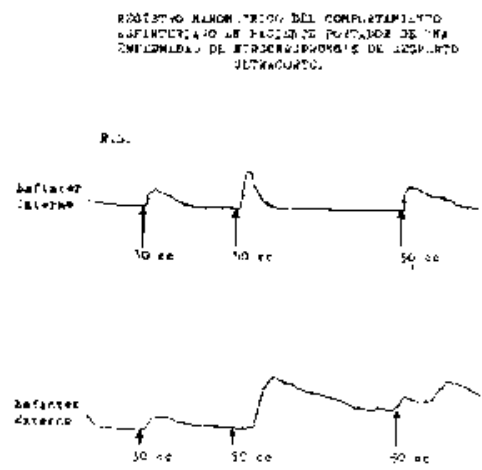

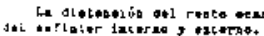

Lizs biopsias que muestran una coloración moderada (café claro) en el tejido muscular y en algurıas fibras nerviosas de la mucosa, pero sin teñir la lámina propia, son consideradas negativas. En cambio, aquellas en las cuales la túnica propia y la muscular de la mucosa aparecen intensamente teñidas (café oscuro) demostrando actividad enzimática muy aumentada, son catalogadas como positivas. (Figuras 3 y 4 ).

La muestra biópsica para estudio histológico clásico se obtiene durante la intervención quirürgica a la que se somete al niño con el diagnóstico confimado de aganglionosis congénita. 


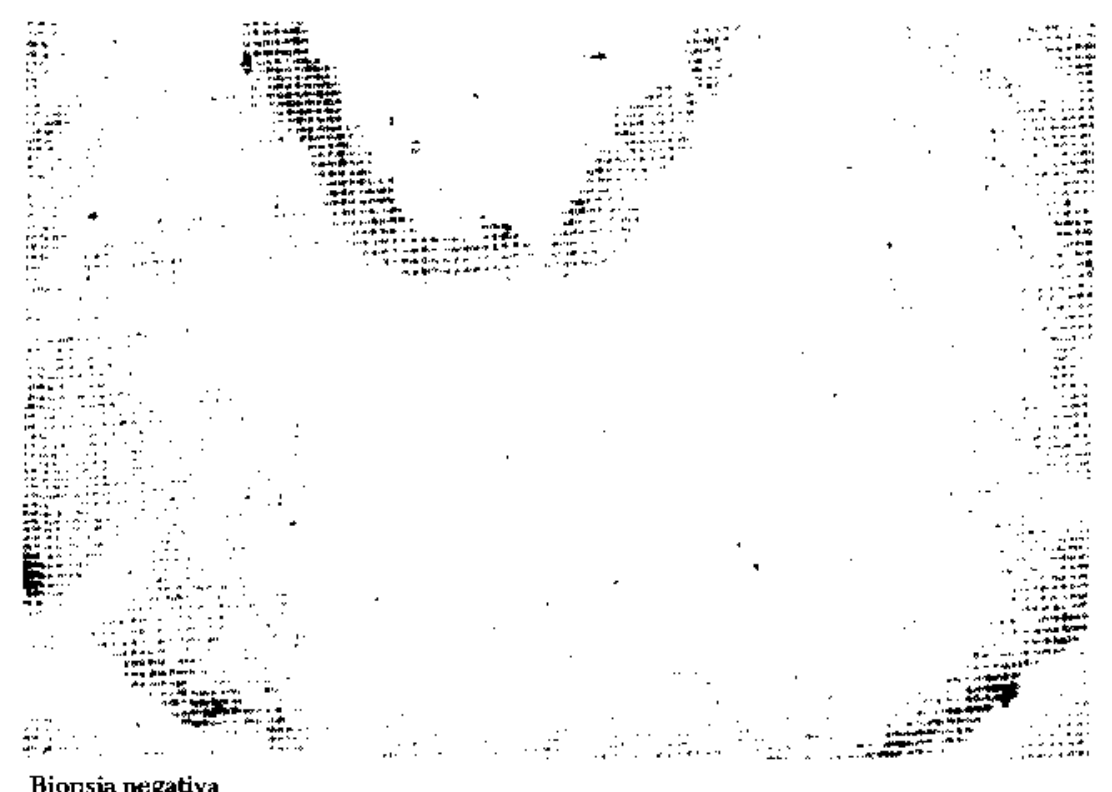

Figura 4

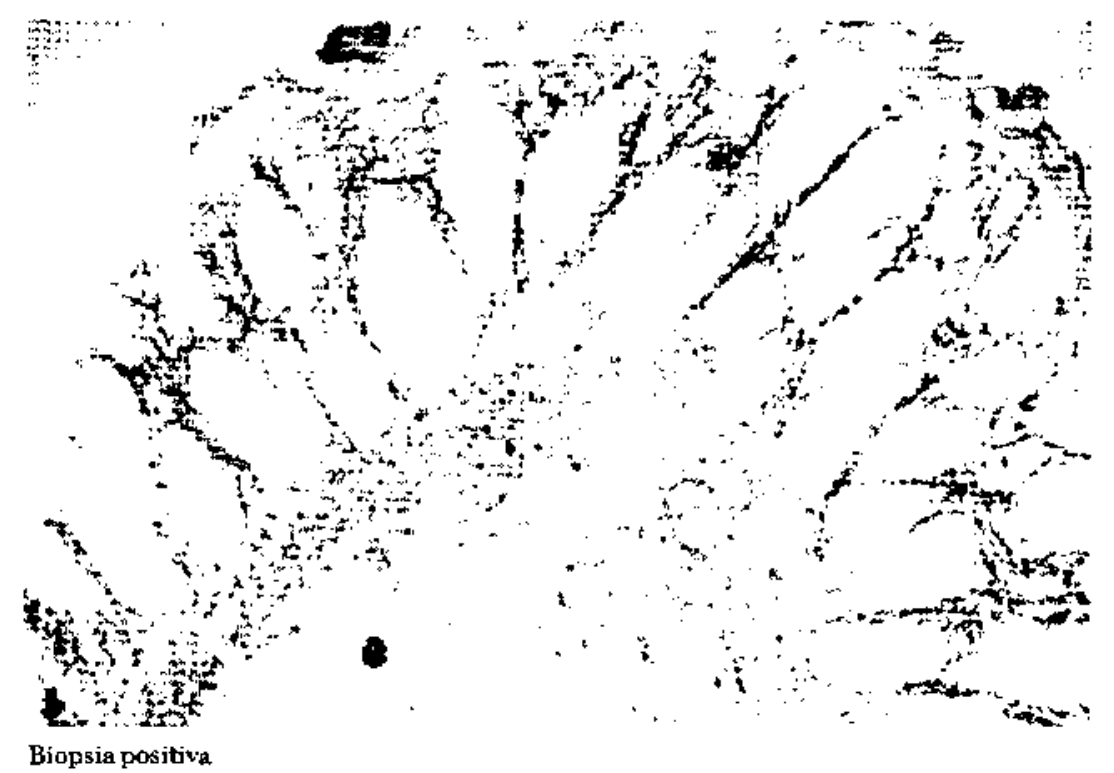

\section{RESULTADOS}

Los 10 niños sanos que constituyen el grupo control para las manometrias, tienen valores de presión basal promedio en el esfínter intemo de $43 \mathrm{~mm} \mathrm{Hg}$, con un rango de 30 a $49 \mathrm{~mm} \mathrm{Hg}$. (Tabla 1 A).

Los 12 niños operados por megacolon congénito constituyeron el gnupo control para el estudio histoquímico. Siete de ellos tenían actividad acetilcolinesterásica altamente positiva y corresponden a niños sometidos a esfinteromiomectomía, en las cuales la muestra biópsica proviene del asa agangliónica. Los otros cinco, con biopsias negativas, correspondian a pacientes operados con técnica de Duhamel, en los que la muestra se obtiene de un asa descendida, con inervación nonmal ( $T a-$ bla 1 B). 
Tabla 1 A

Correlación manométrica, histolígica e histosłuimica cn el estudio del megacolon en el niño.

Grupo (-1)utrol para masumetrias

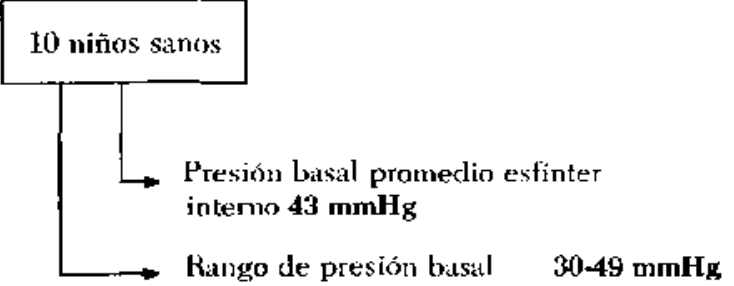

Tabla 1 B

Correlación manométrica, histológica e histoquínicca en el estudio del megacolon en el nin̄o.

Grupo conteol para biopsias histoguímians

12 niños operados de megacolon

-7 con esfinteramionectoría $\longrightarrow$ biopsias

- 5 con técnica de Duhamel $\longrightarrow$ biopsias (Asa gangliónica descendida)

El grupo de 96 pacientes que se sometió al examen manométrico, tenia edades que fluctúan entre 5 meses y 10 años, dividiêndose en 17 lactantes, 30 pre-escolares y 49 escolares (Tabla 2).

\section{Tabla 2}

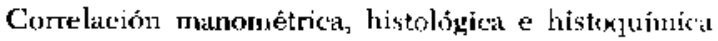
en el estudio de megacolon en el niñes.

Manometrías rectales

Distribución por edad: total 96 siños

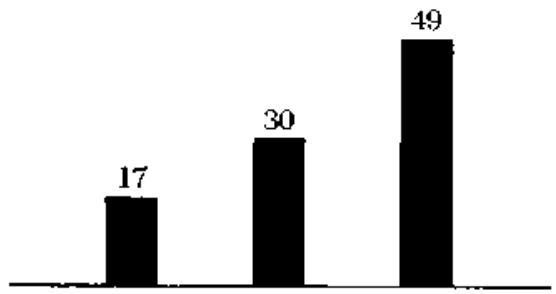

Lactintes Picescolar Escolates
De estos 96 niños, 78 tenían manometrias normales y fueron divididos en dos grupos.

- 48 que se catalogaron como Dolicomegasigmas, presentaban valores de presión basal promedio de $45 \mathrm{~mm} \mathrm{Hg}$, desviación standard (D.S) de 19.6 y rangos de presión entre 20 y $79 \mathrm{~mm} \mathrm{Hg}$. - 30 constipados crónicos refractarios a tratamiento médico y con estudio radiológico sugerente de megacolon funcional, tenian valores de presión basal promedio de $39 \mathrm{~mm}$. D.S. de 14 y rangos de presión entre 30 y $60 \mathrm{~mm} \mathrm{Hg}$ (Tabla 3 ).

Tabla 3

Correlación manometriça, histológica e histoxulumica en el estudio clel megacolon en el niño.

Valores de presion basal

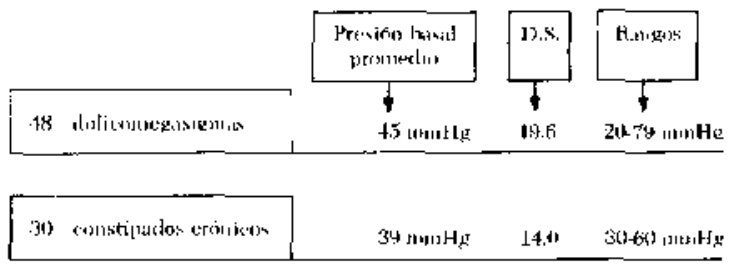

19 :n:ulelunosis

En 12 de los 78 pacientes con manometria normal se hizo el estudio histoquímico para acetilcolinesterasa, resultando en todos negativo.

Estos nin̄os han seguido controlándose en la Policlinica de Gastroenterologia Infantil, y evolucionando favorablemente con tratamiento médico, lo que apoya el diagnóstico de megacolon funcional (Tabla 4).

Tabla 4

Correlación mamométrica, histológica e historduímica en el estudio del megacolon en el niño.

Relacinn entre mitnométricas y biopsias

78 manometrias nonmales

Los 18 pacientes restantes mostraban registros manométricos alterados, con valores de presión 
basal entre 40 y $87 \mathrm{~mm} \mathrm{Hg}$, promedio de $54 \mathrm{~mm}$ y desviación standard 10.7.

Dieciséis de ellos presentaron respuesta paradojal o no respuesta del esfínter interno a la dilatación rectal, y sus biopsias histológicas demostraron claramente aganglionosis congénita; 12 presentaban además una intensa actividad acetilcoljnesterásica en las biopsias histoquímicas.

Por último, en dos niños las manometrias no fueron concluyentes, En uno de ellos, la actividad acetilcolinesterásica intensa y la biopsia histológica alterada certificaron el diagnóstico de aganglionosis. En el otro caso, los estudios histoquimicos positivos en distintos niveles del intestino y la técnica de la deshidrogenasa succínica precisaron el diagnóstico de displasia neuronal colónica; este niño fue sometido a una extirpación total del colon y está actualmente en buenas condiciones (Tabla 5).

\section{Tabla 5}

Correlación nationétrica, histológica e histroxuímica en el estutio del megacolon en el niño.

Relación entre mannnetrías y biopsias

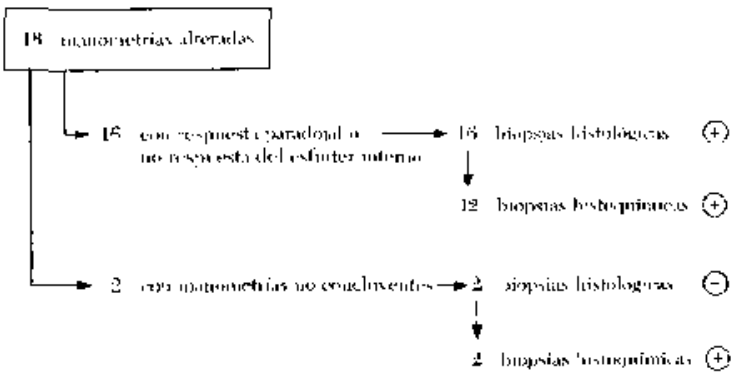

\section{DISCUSION}

El análisis presentado se refiere a un grupo de pacientes relativamente seleccionado, con diagnóstico bien fundamentado de megacolon, controlado en la Policlínica de Gastroenterología Intantil del Hospital Gmo. Grant B. de Concepción, o derivado desde Cirugía Infanti] (Hospital L. Mascayano) para estudio pre-operatorio.

La correlación entre las manometrías rectales efectuadas en condiciones basales y los métodos más agresivos, pero también más precisos, como sor las biopsias quirúrgicas para histología clásica y las biopsias por succión para estudio histoquímico de acetilcolinesterasa, en los casos de megacolon congénito es de un $96 \%$ en nuestro es- tudio, lo que estaría de acuerdo con las referencias revisadas.

La manometría es un examen que no pierde vigencia, fácil de realizar e inocuo, que al resultar normal evita efectuar biopsia.

La biopsia rectal por succión es un método poco agresivo, que no requiere hospitalización, no produce complicaciones, $y$ en manos de un experto histoquímico es totalnıente segura y específica de enfemedad de Hirschspneng.

Es evidente que estos métodos de estudio son técnicas específicas, que sólo pueden realizarse en centros especializados, y que requieren de un grupo de profesionales que trabajen en equipo, el cual debe estar integrado por Pediatra, Gastroenterólogo, Cirujano Infantil, Fisiólogo, Bioquímico y Radiologo.

\section{RESUMEN}

Se practicaron 96 manometrías anorrectales en niños con megacolon. En dieciséis los registros eran compatibles con Enfenmedad de Hirschsprung: en 12 la biopsia rectal por succión para determinar acetilcolinesterasa corroboró el diagnóstico de aganglionosis. En 2 pacientes con manometrías no concluyentes, la actividad acetilcolinesterásica resultó altamente positiva, y en uno de ellos se obtuvo el diagnóstico de displasia neuronal colónica mediante el estudio de la deshidrogenasa succínica.

\section{AGRADECIMIENTOS}

Los autores agradecen al Dr. Bruno Fadda C. por su colaboración en el afinamiento de la técnica histoquímica.

\section{REFERENCIAS}

${ }^{1}$ Denny-Brown and Robertsan E.G. An investigation of the nervous control of defecation. Brain 58, 256-310. 1935.

${ }^{2}$ Schuster M, Hendrix TR and Mendatoff A. The internal anal sphincter response: Manometric studies on its nomal physialogy, neural pathways and alteration in bowel disorders. J. Clin. Invest. 42, 196-207. 1966.

${ }^{3}$ Schuster M.M. Hoomm P. Hendrix TR and Mendeloff A.I Simultaneous manometric reconding of internal and external anal shincter reflexis Bull Johns Hopkins Hosp. 116: 79-88 (1965).

${ }^{4}$ Schoufer L. Talbert LL. and Halker J.A. Differential Sphinctenic Studies in the diagnosis of anorectal disorders of childhood. J. Pediat. Surg. Yol. $2 N^{0} 6,538-543$ (XII - 1967).

5 Meunier P. Mollow P. and Jombert de Beasleu M. L'exploration Manometrique de l' appareil anorectal de l' enfant: application dux megacolon. Pediatric 29, 679686 (1974). 
${ }^{6}$ Danus O, Lamuin F. 1975 Manometria rectal en niño. Trabajo presentado a Jomedos Nacionales de Pediatra. (Santiago, Noviembre 1975 ).

${ }^{7}$ Meunles P. Molland P. and Ronechal I.M. Estudie Manometrique de L' activité reflexe recto anale. J. Physiol (Paris) 69, $276 \mathrm{~A}$, 1974.

${ }^{8}$ Lowson J.ON and Nboon H. Anal Canal pressures in the diagnosis of Hirschprungs disease (1967).

"Schnmfer Loutise. Hirschprumg's Disease Ped. Clin. of N. A. Vol 56, 349-359, 1976.

${ }^{10}$ Meunier P, Rollord P. and Jauber de Beoujal Manometric studies of anorectal disonders in infancy and childhood: An investigation of the physiopathology of continence defacation. B.J. Surg. Vol 63, 402-407, 1976.

11 C. Mortines-Amoyne, R; M. Claver, J. Monerco y F. Corkeres Diagnóstico histoquímico de biopsias por succión en la enfermedad de Hirschsprung. Hev, Clinica Española, Tomo 150 $N^{\circ} 3-4$, pp. 167-171, 1978.

12 Dr. Bruno Fodda C. Fundamentos del diagnóstico histoquímico de la Enfermedad de Hirschsprung. Rev, Chil. Ped, Vol $47 \mathrm{~N}^{0}$ 2, pp. 155-159, 1976.

13 M Kekomait, J. Rapolo, J. Louhimo. Diagnosis of Hirschsprung's disease. Acta Pediatr. Scand. 68: 803-97, 1979.

14 W. Chow, W.C. Chm and Poul C.K Yul (Hong-Kong). Histochemical criteria for the diagnosis of Hirschspnung's disease in rectal suction biopsies by acetylcholinesterase activity. J. Pediatr. Surg., Vol 12 N ${ }^{\circ}$, 675-679, Oct. 77 .

15 W. Meler Rage, P.M. Luttembeic, B. Herng, R. Morger, R. Moses and
A. Schörit. J. Acetylcholinesterase activity in suction biopsies of the sectum in Diagnosis of Hirschsprung's disezse. Pediatr. Surg., Val $7 \mathrm{~N}^{\circ}$ 1, pp. 11-17, Febr., 1972.

${ }^{10}$ Elso Okamoto and Taladed Llada. Embryogenesis of Intramural ganglia of the gut and its relation to Hirsehsprung's disease. J. Pediatr. Surg., Vol 2, 437-443, 5 Oct, 1967.

${ }^{17}$ Compbell and Heler R Nablet. Experience with rectil suction biopsy in the diagrosis of Hirschsprung's disease. P/E. J. Pediatr. Surg, Vol 4, 410-415, 4 August, 1969.

18 J. Smith G. Bestey and W. Parkck Acetylcholinesterase in Hirschsprung"s disease. Lancet 1 (8117): 685, Marzo 79.

${ }^{19} \mathrm{G}$. Dole et als; Diagnostic Value of rectal mucosal acetylcholinesterase levels in Hirschsprung's disease. Lancet 1 (8112): $34 \bar{f}$, Febr., $7 \mathbf{7}$.

$20 \mathrm{~J}$. demet, E. Howard, H. Nixon Histachemical diagnosis of Hirschsprung's disease. Lancet 2 (7617): 436 Ag. 69.

${ }^{91}$ Ebeshand Passonge The genetic of Hirschsprung's disease. New Engl. J. Med., 276: 138, 1967.

22 Hirschsprung's disease: Scandinavian Association of Paediatric, Surgeons, "Spanish Scandinavian Meeting". Acta Paediat. Scand, 63: 307-319, 1974.

23 J.R. Gare, ER. Hownurdand H.H. Nbon. Autonomic Nerves in Rectum and colon in Hirschsprung's Disease. A cholinesterase and catecholamine histochemical study. Arch., Dis., Childn., 44: 406, 1969.

${ }^{24}$ J.P. Elema Hirschspnung's disease: Experience with some enzyme histochemical techniques. Arch., Dis., Childn., 48: 160, 1973. 